\title{
Prevalence and Risk Factors for Dyskinesia among Filipino Patients with Parkinson's Disease
}

\author{
Leah L. Shiong Shu ${ }^{1}$ and Roland Dominic G. Jamora ${ }^{1,2,3}$ \\ ${ }^{1}$ Department of Neurosciences, College of Medicine and Philippine General Hospital, University of the Philippines Manila \\ ${ }^{2}$ Movement Disorders Service and Section of Neurology, International Institute for Neurosciences, \\ St. Luke's Medical Center, Quezon City and Global City, Philippines \\ ${ }^{3}$ Philippine Movement Disorder Surgery Center, Cardinal Santos Medical Center, San Juan City, Philippines
}

\begin{abstract}
Background. Dyskinesia is a drug-induced impairment in the ability to control movements, characterized by repetitive spasmodic motions with lack of coordination. It is usually associated with long term levodopa (Idopa) use brought about by fluctuations in Idopa plasma levels.
\end{abstract}

Objectives. To determine the prevalence and time to dyskinesia among Filipino patients with Parkinson's disease (PD) given Idopa versus dopamine agonist (DA) and identify the risk factors for dyskinesia among patients on Idopa.

Methods. We reviewed the chart of all Filipino patients diagnosed with idiopathic PD seen at the out patient neurology clinics of the Philippine General Hospital and the private clinics of a consultant (RDJ) from 2004-2011. We included patients on monotherapy with either Idopa or DA alone for at least 2 years.

Results. A total of 367 patients were included. The prevalence of dyskinesia was significantly higher among patients on Idopa compared to those on DA ( $36.11 \%$ vs $0.86 \%, p$ 0.005). At 9 years of treatment, a greater proportion of patients on DA remained free of dyskinesia compared to those on Idopa (99.13\%vs 37.5\%). The DA group had a longer time to dyskinesia at 7 years compared to the Idopa group at 6.25 years ( $\mathrm{Cl} 2-20$ years). Among patients on Idopa, younger age (53.29 vs. $62.37, \mathrm{p}<$ $0.05)$, female sex ( 60.44 vs. $39.56 \%, p 0.006)$, and longer duration of treatment $(6.25$ vs. $3.73, p<0.05)$ were significant risk factors for dyskinesia.

Conclusion. Among Filipino PD patients, dyskinesia was significantly higher among patients on Idopa compared to DA. Patients on DA had a longer time to dyskinesia compared to the levodopa group. Among patients on Idopa, the significant risk factors for dyskinesia were: younger age, female sex, and longer duration of treatment.

\section{Key Words: Parkinson's disease, dyskinesia, levodopa, Filipino}

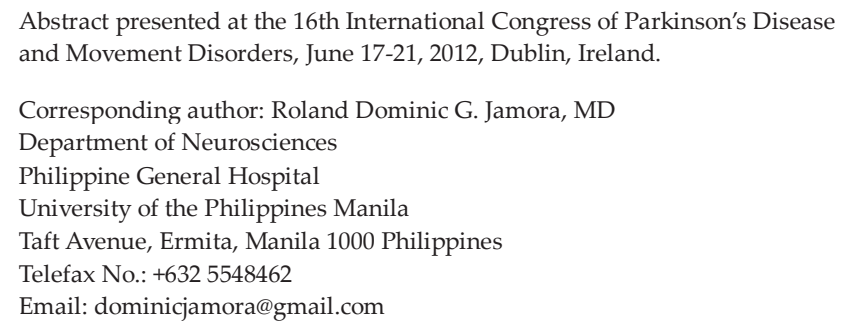

\section{Introduction}

Parkinson's disease (PD) is a condition characterized by resting tremors, rigidity, bradykinesia, and instability brought about by a decrease in dopamine released from the substantia nigra. ${ }^{1}$ As such, the mainstay of treatment for PD is increasing the available dopamine through levodopa (ldopa) or dopamine agonists (DA). ${ }^{1}$ However, the choice of initial treatment for PD remains to be controversial. ${ }^{2}$ Some studies promote the early use of ldopa because of its rapid symptomatic relief. ${ }^{3}$ But other studies encourage the early use of DA to avoid long-term complications of ldopa, such as dyskinesias. ${ }^{4-6}$

Dyskinesia is defined as a drug-induced impairment in the ability to control movements, characterized by spasmodic or repetitive motions with lack of coordination. ${ }^{7}$ Peak-dose dyskinesia manifests as stereotypical choreic or ballistic movements at the height of ldopa levels, while off period dystonia occurs as ldopa levels wear off. ${ }^{7}$ Diphasic dyskinesias consist of periodic episodes of peak dose and off period dyskinesia, and has been correlated with the rise and fall of ldopa plasma levels. ${ }^{8}$ In general, the occurrence of dyskinesia has been associated with prolonged use of high doses of ldopa. ${ }^{9,10}$

The time to occurrence of dyskinesia also varies. In the DATATOP study, dyskinesias were observed in 33\% of patients after 20.5 months of ldopa therapy.11,12 In other studies, the mean time from PD diagnosis to development of dyskinesia was 4 to 6.7 years in $40 \%$ of patients given ldopa. ${ }^{13,14}$ Differences in the study design and the methods used to assess dyskinesia in the clinical setting and in different patient populations accounts for this variability.

The exact mechanism for the development of dyskinesias is still unclear. However, recent studies indicate the importance of pulsatile stimulation of striatal postsynaptic receptors in their pathogenesis. ${ }^{14}$ Data from studies of monkeys treated with 1-methyl-4-phenyl-1,2,3,6tetrahydropyridine have demonstrated that DAs are less likely than ldopa to induce dyskinesia in animals that have not been exposed to ldopa. ${ }^{15}$

To the best of our knowledge, there has been no local data on the prevalence and timing of dyskinesia among Filipino patients with PD. Thus this study aims to determine 
the prevalence of dyskinesia among Filipino patients with PD given ldopa versus DA. It also aims to determine the clinical profile, risk factors, and time for the development of dyskinesia among Filipino PD patients given ldopa and DA.

\section{Methods}

We reviewed the chart of all Filipino patients diagnosed with idiopathic PD seen at the out patient neurology clinics of the Philippine General Hospital and the private clinics of a consultant (RDJ) from 2004-2011. We included patients on monotherapy with either ldopa or DA alone for at least 2 years. These patients should have been on only one type of medication during the observation period. PD patients who underwent surgical treatment were excluded from the study. Demographic data, disease- and treatment-specific variables were collected. The time to the appearance of dyskinesia among patients on ldopa or DA were compared. The risk factors which predisposed the patients to dyskinesia within the ldopa group was then analysed. This study has been approved by the Ethics Review Board of the Philippine General Hospital.

Quantitative variables were expressed as means \pm SD while qualitative variables were expressed as percentages. The $\odot^{2}$ test was used in comparing qualitative variables and the Student's $t$-test for comparing means between groups. Survival curves were plotted using the Kaplan-Meier survival analysis for events (dyskinesia). The log-rank test was used to compare the rate of events between PD patients on ldopa and DA. The data was analysed using SPSS ver 11.5 (SPSS Inc.) and STATA ver 8.0 with a significant $p$ value set at $<0.05$.

\section{Results}

A total of 367 PD patients were included in the study. 252 patients were on ldopa and 115 were on DA as initial treatment. Among those given DA, 55 patients were given pramipexole, 42 were given piribedil, and 18 were given ropinirole. The average age at onset of PD was significantly lower $(53.32 \pm 9.63$ years $)$ in patients given DA compared to those given ldopa $(59.09 \pm 12.9$ years $)(p<0.005)$. The male to female ratio and the baseline Hoehn \&Yahr (H\&Y) score were comparable between the 2 groups (Table 1 ).

Only 1 patient from the DA group developed dyskinesia compared to 91 patients in the ldopa group $(0.87 \%$ vs. $36.11 \%, \mathrm{p}<0.005$ ) (Table 2). Dyskinesia was shown to develop earlier in the ldopa group at 6.25 years [Confidence Interval (CI) 2 - 20 years] compared to the DAgroup (7 years). The cumulative dyskinesia-free rate for the ldopa group was $65 \%$ at 5 years and $37.5 \%$ at 10 years (Figure 1). This was significantly lower than the $99.13 \%$ cumulative dyskinesiafree rate in the DA group.

Among patients on ldopa, the following factors significantly increased the risk for dyskinesia: female sex ( $p$
$0.006)$, younger age $(\mathrm{p}<0.05)$, and longer duration of treatment $(\mathrm{P}<0.05)$ (Table 3$)$.

Table 1. Demographic profile of patients included in the study

\begin{tabular}{lccc}
\hline & ldopa & DA & p \\
& $\mathrm{n}=252$ & $\mathrm{n}=115$ & \\
\hline Male & $130(51.5 \%)$ & $51(44.3 \%)$ & \\
Age at onset (years) & $59.09 \pm 12.9$ & $53.32 \pm 9.63$ & $<0.0005$ \\
Initial H\&Y score & $2.05 \pm 0.67$ & $1.71 \pm 0.57$ & 0.9016 \\
Average treatment duration (years) & $7.49 \pm 3.82$ & $3.85 \pm 1.64$ & $<0.0005$ \\
Treatment duration & $2-20$ & $2-9$ & \\
(range in years) & & & \\
\hline
\end{tabular}

Table 2. Prevalence and time to dyskinesia in patients given ldopa vs. DA

\begin{tabular}{lccc}
\hline & $\begin{array}{c}\text { ldopa } \\
\mathrm{n}=252\end{array}$ & $\begin{array}{c}\text { DA } \\
\mathrm{n}=115\end{array}$ & P \\
\hline $\begin{array}{l}\text { Number of patients who developed } \\
\text { dyskinesia }\end{array}$ & 91 & 1 & \\
\% dyskinesia & $36.11 \%$ & $0.86 \%$ & 0.005 \\
Years to dyskinesia & 6.25 & 7 & \\
Confidence Interval & $(2-20)$ & 7 & \\
\hline
\end{tabular}

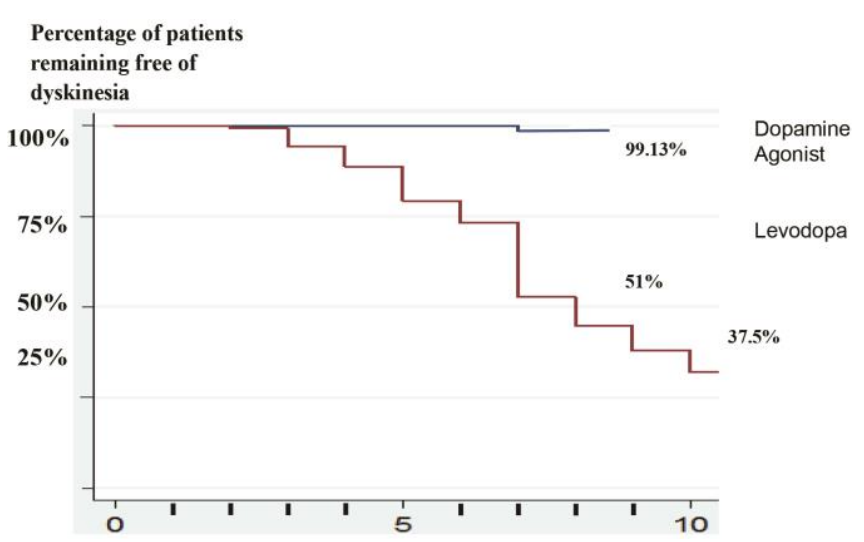

Figure 1. Kaplan Meier Survival Curve of Patients Remaining Free of Dyskinesia in the DA and ldopa Groups

Table 3. Clinical Profile of patients given ldopa who developed dyskinesia

\begin{tabular}{lccc}
\hline \multicolumn{1}{c}{ ldopa Group } & $\begin{array}{c}\text { (+) dyskinesia } \\
\mathrm{n}=91\end{array}$ & $\begin{array}{c}\text { (-) dyskinesia } \\
\mathrm{n}=161\end{array}$ & P value \\
\hline Gender & & & $\mathbf{0 . 0 0 6}$ \\
Male & $39.56 \%$ & $57.80 \%$ & \\
Female & $\mathbf{6 0 . 4 4 \%}$ & $\mathbf{4 2 . 3 4 \%}$ & \\
Age at diagnosis (years) & $\mathbf{5 3 . 2 9 \pm \mathbf { 1 2 . 3 7 }}$ & $\mathbf{6 2 . 3 7 \pm \mathbf { 1 2 . 0 5 }}$ & $<\mathbf{0 . 0 5}$ \\
Initial H\&Y & $2.05 \pm 0.64$ & $2.05 \pm 0.68$ & 0.900 \\
Initial Dose (mg/day) & $454.08 \pm 261$ & $396.35 \pm 249$ & 0.072 \\
Years of treatment & $\mathbf{6 . 2 5} \pm 3.41$ & $3.73 \pm 2.52$ & $<\mathbf{0 . 0 5}$ \\
Maximum dose given & 1200 & 800 & $<\mathbf{0 . 0 5}$ \\
(mg/day) & & & \\
$\begin{array}{l}\text { Dose at dyskinesia } \\
\text { (mg/day) }\end{array}$ & 850.41 & & \\
\hline
\end{tabular}




\section{Discussion}

The male to female ratio in our study subjects is approximately 1:1. This was different compared to studies done in the US and Canada, where the incidence of PD was approximately twofold higher in men than in women. ${ }^{16}$ Two other studies of PD patients done in China and Taiwan also showed that, as opposed to the higher incidence of PD in male patients in western countries, the male:female ratio in Asian PD patients is 1.0 to $1.2 .17,18$

In Western studies, the mean age at diagnosis range from 61 - 70 years, ${ }^{16}$ while it was 57 years old in our study population. When we compared the mean age of patients between the treatment groups, the patients in the DA group were significantly younger than those in the ldopa group (59.09 years vs. 53.32 years). This might have reflected the practice of the Filipino neurologists of using DA for younger patients to decrease the complications of long term use of ldopa. ${ }^{19}$

Among all our patients, the average $H \& Y$ score at diagnosis of PD is 1.98. This was similar to a study done in 2004, which showed that the average H\&Y score at diagnosis of PD was 2.0. ${ }^{20}$ When we compared the initial H\&Y score between treatment groups, the DA group had lower H\&Y scores compared to the ldopa group (1.71 vs. 2.05, p 0.91), but this was not significant.

There were more patients in the ldopa group who developed dyskinesias. These findings imply that compared to DA, treatment with ldopa significantly increases the development of dyskinesia among patients with PD. This data was similar to previous trials which also showed increased incidence of dyskinesia in patients given ldopa compared to those given the DA (cabergoline, pergolide, and pramipexole) over 3-5 years of therapy. ${ }^{4,6,19}$ In a prospective study, the incidence of dyskinesia was significantly higher in the ldopa group at $45 \%$ compared to the ropinirole group $(20 \%))^{5,11}$

In terms of time to dyskinesia, Table 2 shows that the average time to dyskinesia was earlier in the ldopa group compared to that in the DA group. There was only 1 patient in the DA group who developed dyskinesia on monotherapy at 7 years. This was a 53-year-old female patient taking piribedil at a total dose of $300 \mathrm{mg} /$ day. The dose of this patient was noted to be too high, as the recommended dose of this drug is only up to $150 \mathrm{mg} /$ day. $^{2}$ The increased dose of piribedil may have contributed to the occurrence of dyskinesia in this patient.

The time to dyskinesia in the ldopa group in our study (34\% at 5 years, $51 \%$ at 7 years) was comparable with the previous studies. A retrospective study showed that slightly less than $40 \%$ of PD patients develop dyskinesia after 4 to 6 years of ldopa treatment. ${ }^{14}$ In a community based study in UK, dyskinesia was seen in $28 \%$ of PD patients 6.7 years after ldopa treatment. ${ }^{13}$ The results were also similar to that of a clinical-pathological study of patients in which $31 \%$ of the patients experienced dyskinesia after 6.4 years of treatment. In a study done by Schrag and Quinn, dyskinesia occurred in $32 \%$ of those treated for 6 to 9 years, and in $89 \%$ of those treated for more than 10 years. ${ }^{13}$ This data suggests that among Filipino PD patients, the anticipated treatment duration of $5-10$ years on ldopa would put them at 51 $62.5 \%$ risk for dyskinesia.

The exact mechanism for the development of dyskinesia is still unclear. However, recent studies have demonstrated the importance of pulsatile stimulation of striatal postsynaptic receptors in the pathogenesis of dyskinesia. The short half-life of ldopa increases its potential to induce intermittent stimulation of dopamine receptors, and fluctuations in dopamine levels in the body, which produces dyskinesia..22 DAs on the other hand, have longer half lives, and are less associated with dyskinesias.

The risk factors associated with dyskinesia among our patients were younger age, female sex, and longer years of treatment (Table 3). This was similar to several western studies. In a study in 1997, age at PD onset is the single most important risk factor for the development of dyskinesia. ${ }^{23}$ In other studies, younger age of onset and female sex were identified as risk factors for dyskinesia. ${ }^{10}$ Two other studies in Asia also showed that longer duration of treatment was a significant risk factor for the development of dyskinesia. ${ }^{13}, 23$

The effects of age, gender, and duration of treatment on the occurrence of dyskinesia have been investigated in several studies. In one study, increased age was associated with diminished response and decreased sensitivity to ldopa therapy. ${ }^{24}$ Younger age, on the other hand is associated with higher sensitivity to ldopa and more fluctuations in serum drug levels. The effect of gender on dyskinesia in PD patients was associated with genetic and weight differences between females and males. Females have been shown to have less genetic protection due to lower expression of the gene for the dopamine receptor D2 and are more prone to develop dyskinesia than male patients. ${ }^{25}$ A lower initial body weight in females translated to a higher ldopa dose per $\mathrm{kg}$ bodyweight compared to male patients. ${ }^{26}$ This led to a higher level of plasma ldopa concentration under the curve when corrected as $\mathrm{mg} / \mathrm{kg}$ body weight, and higher bioavailability of ldopa in female patients. ${ }^{9,27}$

The development of dyskinesia due to longer duration of treatment have been associated with increased exposure to ldopa and the progression of the disease. With advancing disease, the benefit of ldopa diminishes and the threshold for developing dyskinesia decreases. ${ }^{28}$ There is also a higher fluctuation of dopamine levels even with a similar dose of ldopa with increasing severity of PD. ${ }^{29}$ This is likely to be the result of plastic changes and enhanced dopamine sensitivity of D1 receptors in chronic ldopa therapy. ${ }^{30,31}$ The pulsatility of dopamine release becomes more prominent and fluctuations between hypersensitivity and tolerance would lead to dyskinesia. An additional factor for this variable 
response is the continuing neuronal loss predisposing to dyskinesia with ldopa in advancing disease. ${ }^{32}$

This retrospective study has several limitations. The data obtained was limited by the available information from the patient's chart. Thus, other variables such as the UPDRS motor scores was not included due to incomplete charts. Data on dose adjustments were unreliable as most patients adjusted the frequency of medication intake based on the severity of their symptoms. Compliance to medications cannot be assured for all study participants and follow up period was also variable depending on physician preference and patient conformance. Despite this limitation, this was the only study on dyskinesia among Filipino PD patients on ldopa or DA monotherapy.

\section{Conclusion}

Our study showed that the prevalence of dyskinesia (36.11\%) was significantly higher among patients on ldopa monotherapy compared to those on DA. Patients on DA monotherapy had a longer time to the onset dyskinesia at 7 years of treatment compared to those in the ldopa group at 6.25 years (range at $2-20$ years of treatment). Among patients on ldopa, the significant risk factors that predispose patients to the development of dyskinesia were: younger age, female sex, and longer duration of treatment. The identification of these risk factors may aid in the choice of initial treatment, to avoid or delay the development of dyskinesias among patients with PD.

\section{References}

1. Hubble JP. Novel drugs for Parkinson's disease. Med Clin North Am. 1999; 83(2):525-36.

2. National Collaborating Centre for Chronic Conditions. Parkinson's disease: national clinical guideline for diagnosis and management in primary and secondary care. London: Royal College of Physicians, 2006.

3. Cotzias GC, Papavasiliou PS, Gellene R. Modification of parkinsonism chronic treatment with L-dopa. N Engl J Med. 1969; 280(7):337-45.

4. Rascol O, Brooks DJ, Korczyn AD, De Deyn PP, Clarke CE, Lang AE. A five-year study of the incidence of dyskinesia in patients with early Parkinson's disease who were treated with ropinirole or levodopa. N Engl J Med. 2000; 342(20):1484-91.

5. Parkinson Study Group. Pramipexole vs levodopa as initial treatment for Parkinson disease: a randomized controlled trial. JAMA. 2000; 284(15):1931-8.

6. Oertel WH, Wolters E, Sampaio C, et al. Pergolide versus 1-dopa monotherapy in early Parkinson's disease: the PELMOPET study. Mov Disord. 2006; 21(3):343-53.

7. Jankovic J. Motor fluctuations and dyskinesias in Parkinson's disease: clinical manifestations. Mov Disord. 2005; 20 Suppl 11:S11-6.

8. Parkinsons Study Group. L-dopa and the progression of Parkinson's Disease. N Engl J Med. 2004;351(24):2498-2508.

9. Zappia M, Annesi G, Nicoletti G, et al. Sex differences in clinical and genetic determinants of levodopa peak-dose dyskinesias in Parkinson disease. Arch Neurol. 2005; 62(4):601-5.

10. Sharma JC, Bachmann CG, Linazasoro G. Classifying risk factors for dyskinesia in Parkinson's disease. Parkinsonism Relat Disord. 2010; 16(8):490-7.
11. Hauser RA, McDermott MP, Messing S. Factors associated with the development of motor fluctuations and dyskinesias in Parkinson disease. Arch Neurol. 2006; 63(12):1756-60.

12. Parkinson Study Group. Impact of deprenyl and tocopherol treatment on Parkinson's disease in DATATOP patients requiring levodopa. Ann Neurol. 1996; 39(1):37-45.

13. Schrag A, Quinn N. Dyskinesias and motor fluctuations in Parkinson's disease. A community-based study. Brain. 2000;123(Pt 11):2297-305.

14. Ahlskog JE, Muenter MD. Frequency of levodopa-related dyskinesias and motor fluctuations as estimated from the cumulative literature. Mov Disord. 2001; 16(3):448-458.

15. Pearce RK, Banerji T, Jenner $P$, et al. De novo administration of ropinirole and bromocriptine induces less dyskinesia than L-dopa in the MPTPtreated marmoset. Mov Disord. 1998; 13(2):234-41.

16. Van Den Eeden S, Tanner C, Bernstein A, et 1. Incidence of Parkinson's disease: Variation by age, gender, and race/ethnicity. Am J Epidemiol. 2003; 157(11):1015-22.

17. Wang YS, Shi YM, Wu ZY, et al. Parkinson's disease in China. Coordinational Group of Neuroepidemiology, PLA Chin Med J (Engl). 1991; 104(11):960-4.

18. Chen RC, Chang SF, Su CL, et al. Prevalence, incidence, and mortality of PD: A door-to-door survey in Ilan county, Taiwan. Neurology. 2001; 57(9):1679-86 .

19. Nutt JG, Wooten GF. Diagnosis and initial management of Parkinson's disease. N Engl J Med. 2005; 353(10):1021-7.

20. Okubadejo NU, Ojo OO, Oshinaike OO. Clinical profile of parkinsonism and Parkinson's disease in Lagos, Southwestern Nigeria. BMC Neurology. 2010; 10(1):1-6.

21. Chase TN, Bibbiani F, Oh JD. Striatal glutamatergic mechanisms and extrapyramidal movement disorders. Neurotox Res. 2003;5(1):139-46.

22. Brotchie JM. Nondopaminergic mechanisms in Levodopa-induced dyskinesia. Mov Disord. 2005; 20(8):919-31.

23. Miyawaki E, Lyons K, Pahwa R et al. Motor complications of chronic Ldopa therapy in Parkinson's Disease. Clin Neuro Pharmacol. 1997; 20(6):523-30.

24. Blin J, Dubois B, Bonnet AM, et al. Does ageing aggravate parkinsonian disability? J Neurol Neurosurg Psychiatry. 1991; 54(1):780-2.

25. Aubert I, Guigoni C, Hakansson K, et al. Increased D1 dopamine receptor signaling in Levodopa-induced dyskinesia. Ann Neurol. 2005; 57(1):17-26.

26. Bachmann CG, Zapf A, Brunner E, Trenkwalder C. Dopaminergic treatment is associated with decreased body weight in patients with Parkinson's disease and dyskinesias. Eur J Neurol. 2009; 16(8):895-901.

27. Arabia G, Zappia M, Bosco D, et al. Bodyweight, levodopa pharmacokinetics and dyskinesia in Parkinson's disease. J Neurol Sci. 2002; 23(Suppl 2):S53-4.

28. Mouradian MM, Juncos JL, Fabbrini G, et al. Motor fluctuations in Parkinson's disease. Ann Neurol.1989; 25(1): 437-78.

29. dela Fuente-Fernandez RD, Sossi V, Huang Z, et al. Levodopa induced changes in synaptic dopamine levels increase with progression of Parkinson's disease: implications for dyskinesia. Brain. 2004; 127(12):2747-54

30. Calabresi P, Di Filippo M, Ghiglieri V, Picconi B. Molecular mechanisms underlying levodopa-induced dyskinesia. Mov Disord. 2008; 23(Suppl 3):S570-9.

31. Nutt JG, Chung KA, Holford NH. Dyskinesia and the antiparkinsonian response always temporally coincide: a retrospective study. Neurology. 2010; 74(15):1191-7.

32. Onofri M, Paci C, Thomas A. Sudden appearance of invalidating dyskinesia-dystonia and off fluctuations after the introduction of 1-dopa in two dopaminomimetic drug naive patients with stage IV Parkinson's disease. J Neurol Neurosurg Psychiatry. 1998; 65(12):191-5. 